\title{
SKIN CLOSURE USING SIMPLE INTERRUPTED AND CONTINUOUS SUBCUTICULAR NYLON SUTURES: A COMPARISON OF RESULTS
}

\author{
Loo JT and Md Razif MA \\ Department of Orthopaedic Surgery, University of Malaya Medical Centre, 50603 Kuala Lumpur
}

\begin{abstract}
Skin closure using simple interrupted nylon sutures was compared with closure using subcuticular nylon sutures in $\mathbf{8 0}$ consecutive patients undergoing semiemergency surgery, involving open reduction and internal fixation of either the forearm bones or femur. The simple interrupted technique was shown to be slower than the subcuticular technique with higher early postoperative wound complication rate. It may use an extra packet of sutures particularly if the average wound length is $19.8 \mathrm{~cm}$. There is, however, no statistical difference demonstrated for the late scar complaints or subjective and objective scoring of cosmetic outcomes six months after the surgery. (JUMMEC 2003-2005; 8: 56-60)
\end{abstract}

KEYWORDS: Simple interrupted, subcuticular, skin closure, nylon, cosmetic outcome

\section{Introduction}

The skin as a barrier between the internal structures and the external environment is exceptionally susceptible to injury, either through accidental injury or planned surgical incision. Today, as surgery increases in complexity, and the heightened public awareness of scar cosmesis and skin healing need to be optimized to ensure the overall success of the surgery. The technique of suturing which is a method of closing wounds is thousands of years old. Although suture materials and techniques have changed, the goals remain the same: closing dead space, supporting wounds until healing increases their tensile strength, approximating skin edges for an aesthetically pleasing and functional result, and minimizing the risks of bleeding and infection.

A number of studies published have discussed the various aspects of the wound closure (I-5). However, direct comparison between different suturing techniques is lacking. There is also no standardized protocol for the methods, lack of agreement in the outcome measurement and scoring system. In general, simple interrupted technique of wound closure is commonly performed, as it is easy to learn and master. However, it is thought to be time-consuming with high complication rate and inferior cosmetic result. On the other hand, subcuticular technique is considered an elegant but difficult suturing technique. Running subcuticular closure is also time-consuming and does not evert wound edges well. It may show superior cosmetic result in certain locations of the body. Many studies have been performed on general surgical procedures, a number of which were often low tissue trauma and short incisions $(I-4,6,7)$. Orthopaedic trauma surgical procedures have the following features. Firstly, the surgery is complicated by preexisting and extensive soft tissue injury. Secondly, the dissection needs to be deep and must reach the bone before any meaningful internal fixation could be done. There is relatively rough tissue handling and surgical trauma, particularly during the implants insertion. Thirdly, the presence of the implants increase the tissue tension and this is worse if the bulky plate is used on the thin patients. The skin is subjected to excessive distraction force. Last but not least, the surgery is usually time-consuming with lengthy wounds. Therefore, a randomized prospective study was carried out to examine the advantages and disadvantages of the two commonest suturing techniques, that is, simple interrupted and continuous subcuticular, in closing the wound in clean orthopaedic surgery. Attempt is made to compare the short-term outcome of the cosmetic result between the suturing techniques, which is seldom mentioned in most previous publications due to the very subjective nature of this parameter. 


\section{Methods}

Eighty consecutive healthy patients with either closed fracture of radius-ulna or femur operated between September 200I and March 2002 were included in this study. The patients were considered for the study if they were above 12 years old, sustained isolated closed fracture of radius-ulna or femur and treated by open reduction and internal fixation with metallic implants. The following patients would be excluded: those with open fracture, personal or family history of keloid or hypertrophic scar formation, presence of abrasion, previous surgery and preexisting skin disease at the operating site, medical conditions for example, steroid usage, diabetes mellitus, blood clotting disorder, peripheral vascular disease and haemoglobin level less than $100 \mathrm{~g} / \mathrm{l}$. The patients were randomized into two groups, simple interrupted and continuous subcuticular groups. To reduce the inter-surgeon variability during the surgery, only the medical officer in the Masters program third year and above, were performing the final wound closure. To further reduce the variation, a handout of protocol was distributed to each of the medical officers involved. At the operation theatre, the starting time of the surgery was recorded once the skin incision was made. The fracture site would be identified. The fracture was mobilized, reduced and stabilized. At the end of surgery, the wound was cleaned with copious amount of normal saline. For both groups, the deep fascia was closed continuously with polyglactin 910 size 1 or 0 , and dermal layer was closed with polyglactin 910 size $2 / 0$ or 3/0 with simple interrupted method. Depending on the randomization, the final skin closure would be either simple interrupted or continuous subcuticular techniques, using nylon $3 / 0$ or $4 / 0,3 / 8$ c, reverse cutting, (75 cm in length, Ethilon; ethicon).

The patients were followed up on day 14, 3rd month and 6th month postoperatively. Early wound complications and late scar complications were recorded. At the 6th month follow-up, the cosmetic outcome was assessed. These included subjective assessment by the patient and assessment by the author using modified clinical scar scoring (8). Digital photographs of each scar were also taken in the standardized fashion throughout the study period for later assessment by a panel of medical personnel who were blind to the study.

\section{Results}

\section{Demographic data}

A total of 80 patients were available. At subsequent visits, the number of patients declined gradually as some of them defaulted the follow-up. The surgery to close the wound was performed by different surgeons of varying skill levels. To eliminate the influence of operator variability, the number of simple interrupted data entries from a given surgeon must equal his subcuticular data entries. In cases in which the data did not meet this criterion, data from the larger group were selected in a random manner, so that both groups would have equal number of entries for that particular surgeon.

For this reason, only 76, 66 and 59 patients at day I4, 3 rd month and 6th month respectively were available for analysis (Table I).

In Table 2, both simple interrupted and continuous subcuticular groups were comparable with no significant difference at different time interval for the following parameters: number of patients, age, sex and racial distribution and smoking status. No significant difference was found between the groups in terms of mode of injury, number of day spent in the hospital before and after the surgery, duration of the surgery, the length of the wound at different time interval, sites of surgery and types of implants used.

\section{Clinical outcomes}

For comparison purposes, the time taken to close the wound was divided by the length of the wound. Subcuticular closure was accomplished at a significantly faster rate (mean $35.66 \mathrm{sec} / \mathrm{cm}$ ) than simple interrupted closure $(46.84 \mathrm{sec} / \mathrm{cm})(\mathrm{t}=3.56, p=0.00 \mathrm{I})$.

Most of the wounds required only one packet (75 $\mathrm{cm} /$ packet) of suture to close the wound. However, four out of 38 patients in the simple interrupted group needed extra packet of suture. All wounds in the subcuticular group were accomplished with single packet of suture. None of the patients in either group needed more than two packets of suture to close the wound. Further analysis showed that the simple interrupted group that needed one and two packets to close the wound, the average length of the wound was 10.7 and $19.8 \mathrm{~cm}$ respectively.

The wounds closed with subcuticular technique showed a significantly lower frequency of postoperative complication, for example, tissue reactivity, gapping infection etc., compared to the simple interrupted group $(p=0.004)$.

However, at the 3rd month and 6th month postoperatively both groups had almost equal number of patient who developed complications of the scar. There was no significant difference between the groups. 
Table I. Number of patients

\begin{tabular}{|c|c|c|c|c|}
\hline & At Beginning & Day 14 & 3rd Month & 6th Month \\
\hline Defaulters & - & 0 & 8 & 7 \\
\hline Patients available & 80 & 80 & 72 & 65 \\
\hline $\begin{array}{l}\text { *Actual number of patients used for } \\
\text { analysis (after randomized selection) }\end{array}$ & 76 & 76 & 66 & 59 \\
\hline
\end{tabular}

*The patient was selected in a randomized manner so that the number of simple interrupted data entries from a given surgeon equaled his subcuticular data entries.

Table 2. General characteristics of the patients at various time intervals

General characteristics of the patients according to the group at the beginning and day 14 of the study

\begin{tabular}{lccc}
\hline & Simple Interrupted & Subcuticular & Statistic \\
\hline Number of patients & 38 & 38 & No difference \\
Age, $(y r s \pm$ sd) & $42.26 \pm 20.63$ & $45.11 \pm 23.34$ & No difference \\
Sex, Male:Female & $26: 12(68.4 \%: 31.6 \%)$ & $25: 13(65.8 \%: 34.2 \%)$ & No difference \\
Race, Malay:Chinese:Indian:Others & $12: 13: 10: 3$ & $14: 11: 11: 2$ & No difference \\
Smoker:Non-smoker & $7: 31$ & $9: 29$ & \\
\hline
\end{tabular}

General characteristics of the patients according to the group at the 3rd month of the study

\begin{tabular}{lccc}
\hline & Simple Interrupted & Subcuticular & Statistic \\
\hline Number of patients & 32 & 34 & No difference \\
Age, $(y r s \pm s d)$ & $41.28 \pm 19.64$ & $42.74 \pm 22.43$ & No difference \\
Sex, Male:Female & $24: 8(75 \%: 25 \%)$ & $22: 12(64.7 \%: 35.3 \%)$ & No difference \\
Race, Malay:Chinese:Indian:Others & $10: 10: 10: 2$ & $13: 9: 10: 2$ & No difference \\
Smoker:Non-smoker & $6: 26$ & $9: 25$ &
\end{tabular}

General characteristics of the patients according to the group at the 6th month of the study

\begin{tabular}{lccc}
\hline & Simple Interrupted & Subcuticular & Statistic \\
\hline Number of patients & 31 & 28 & No difference \\
Age, $(y r s \pm$ sd $)$ & $41.74 \pm 19.79$ & $43.86 \pm 23.26$ & No difference \\
Sex, Male:Female & $23: 8(74.2 \%: 25.8 \%)$ & $18: 10(64.3 \%: 35.7 \%)$ & No difference \\
Race, Malay:Chinese:Indian:Others & $9: 10: 10: 2$ & $10: 7: 9: 2$ & No difference \\
Smoker:Non-smoker & $6: 25$ & $7: 21$ &
\end{tabular}

Similarly, both groups showed no significant difference in the cosmetic outcomes whether this was assessed by the patients using grading system, visual analogue scale, rating by the author using modified clinical scar scoring or objective scoring by the panel of medical personnel using visual analogue scale.

\section{Discussion and Conclusions}

The study was designed as a randomized prospective study. Every effort was taken to ensure the similarity between the two groups. This was evident when both the simple interrupted and subcuticular groups showed the near equal if not perfect result in the demographic and clinical features.

For the first parameter, that is, speed of wound closure, after eliminating the influence of the length of wound on the closure time, the subcuticular closure was accomplished at a faster rate compared to the simple interrupted group. The reason this difference exists most likely due to the following factors:

- Time was spent while waiting for the assistant to cut the suture in the simple interrupted method. In the subcuticular method, the suture was only cut once, that is, at the end of skin closure. 
- The needle was much nearer to the wound before the start of next stitch in the subcuticular group compared to the simple interrupted group.

- Extra time spent on tying the multiple knots in the simple interrupted closure, whereas there was no such requirement for the subcuticular method.

For the second outcome measured, almost all the wounds were closed with a single packet of suture. A few wounds in the simple interrupted group needed two packets of sutures to complete the closure. None in the subcuticular group needed two packets of suture. Analysis showed that the average length of wound for those who needed two packet of sutures was $19.8 \mathrm{~cm}$, as compared to $10.7 \mathrm{~cm}$ for those whose wound closed with single packet of suture in the simple interrupted group. The tendency for the extra packet of suture in the simple interrupted group can be explained by the following facts:

- By the nature of the technique, some length of suture was left behind for the ease of removal. Typically about $\mathrm{I}-2 \mathrm{~cm}$ of the suture was used for this purpose. The length would vary among the surgeons.

- Surgeons differ in their ability to secure a knot with a shorter length of suture. Some surgeons were much more comfortable to tie a knot with excessive length of suture. This would be a waste, as the excess suture would be removed after completion of the knot. No such waste would ever occur in the subcuticular closure, as no knot tying is necessary except at the end of the closure.

- At the very end of suture, it is very difficult to utilize fully the short remnant of the suture attached to the needle.

Subcuticular closure was not necessarily more economical. Frequently the subcuticular closure could not oppose the wound well, leaving a mildly gapped wound at multiple loci. This was commonly treated with secondary reinforcement methods, for example, steristrip. The overall economic cost resulting from the use of this additional reinforcement would have equaled the cost of the extra packet of suture.

The simple interrupted group showed higher incidence of early wound complication as compared to subcuticular group. Most of the complications were due to the development of tissue reactivity. The overall incidence of wound infection in this study was about $2.5 \%$, which is comparable to the worldwide figure quoted for clean surgery (9, I-2). All infected wounds were cleared successfully with daily dressing and fiveday course of antibiotic. Most studies in English literature with similar designs showed no apparent difference in the postoperative wound complication regardless of the technique utilized, for example, hernia and upper abdominal surgery (3), perineal repair (4), and neurosurgical surgery (6). However, it was shown that postoperative sensory recovery in the leg after saphenous vein coronary artery bypass graft was better preserved with the simple interrupted method (10).

Scar complications were comparable in both groups. This showed that the methods of wound closure had no bearing on the long-term scar complaints, either on the $3 r d$ month or 6 th month after the surgery.

This study attempted to compare the cosmetic outcome of the wound closure by providing the subjective and objective assessment. The patients were asked to assess their wounds. It was noted that most patients rated their wounds good or fair, and infrequently graded as poor. Overall, there was no difference between the groups on self-assessment. The assessment of cosmetic result with visual analogue scale allowed translation of a complex subjective experience to a visual-spatial display, which involves perceptual judgment and accuracy. Previous work has shown the minimal clinically important difference on the visual analogue cosmesis scale to be $1.5 \mathrm{~cm}$ (8). This study had sufficient power ( $90 \%$ with $0.05 \alpha$-error) to determine a $1.6 \mathrm{~cm}$ difference on the visual analogue scale. The objective scoring by the patients utilizing visual analogue scale showed no difference in the perception of the cosmetic result of the wound in between the groups.

Difficulties in understanding the visual analogue scale did occur, especially amongst manual worker and older patients. Much time was devoted to explaining the function and how to use the visual analogue scale correctly.

Clinical scar scoring by the author using the modified clinical scar scoring system (8) showed equal results between two groups. This provides an objective way to assess the subjective parameter.

For both the categorical or visual analogue scale, the subjects were asked to score their wound without comparison. They had no idea except from the limited past experience how well the wound should appear. For this reason, a third assessment method was utilized. Three standardized photographs for each patient were taken and assessed by a panel of three medical personnel who were blind to the study, using visual analogue scale. Analysis of the result further showed no difference existed in between the wound closed by the simple interrupted or subcuticular technique. The result of the cosmetic outcomes is 
comparable to the similar design of study in hernia surgery (3) and saphenous vein harvesting in coronary artery bypass surgery (10).

In conclusion, the choice of technique for wound closure did not affect the final outcome of the wound, up to the 6th month after the surgery. Until additional studies are available, the surgeons are free to select the technique of their preference.

\section{References}

I. Gabrielli F, Potenza C, Puddu P, et al. Suture materials and other factors associated with tissue reactivity, infection, and wound dehiscence among plastic surgery outpatients. Plast Reconstr Surg, 200 I; 107:38.

2. McLean NR, Fyfe AHB, Flint EF, et al. Comparison of skin closure using continuous and interrupted nylon sutures. Br J Surg, 1980; 67:633-635.

3. Cassie $A B$, Chatterjee AK, Mehta $S$, et al. Pain quantum and wound healing: a comparison of interrupted inversion PDS and standard nylon sutures in abdominal skin closure. Ann R Coll Surg Engl, 1988; 70(6):339-42.

4. Mahomed K, Grant A, Ashurst H, et al. The Southmead perineal suture study. A randomized comparison of suture materials and suturing techniques for repair of perineal trauma. $\mathrm{Br}$ J Obstet Gynecol, 1989; 96(II): 1272-80.
5. Shamiyeh A, Schrenk P, Steltzer T, et al. Prospective randomized blind controlled trial comparing sutures, tape and octycyanoarylate tissue adhesive for skin closure after phlebectomy. Dermatol Surg, 200I; 27(10):877.

6. Mylonas C, Brocklehurst G. A prospective randomized comparison of continuous Polydioxanone and interrupted Neurilon for neurosurgical wound closure. Br J Neurosurg, 1991; 5:357-360

7. Ouinn JV,Well G. Outcome measures in clinical wound studies: The reliability of cosmesis scales with a determination of a minimal clinically important difference. Acad Emerg Med, 1998; 5:583-586.

8. Dolynchuk KN, Ziesmann M, Serletti JM. Topical Putrescine (Fibrostat) in Treatment of Hypertrophic Scars: Phase II Study. Plast Reconstr Surg, 1996; 97:I 17.

9. Nichols RL. Surgical infection: Prevention and treatment - 1965 to 1995. Am J Surg, 1996; 172:68.

10. Nair UR, Griffiths G, Lawson RA. Postoperative neuralgia in the leg after saphenous vein coronary artery bypass graft: a prospective study. Thorax, I988; 43(I):4I-3. 